\title{
SHAPE MINIMIZATION OF DENDRITIC ATTENUATION
}

\author{
ANTOINE HENROT AND YANNICK PRIVAT
}

\begin{abstract}
What is the optimal shape of a dendrite? Of course, optimality refers to some particular criterion. In this paper, we look at the case of a dendrite sealed at one end and connected at the other end to a soma. The electrical potential in the fiber follows the classical cable equations as established by W. Rall. We are interested in the shape of the dendrite which minimizes either the attenuation in time of the potential or the attenuation in space. In both cases, we prove that the cylindrical shape is optimal.
\end{abstract}

\section{INTRODUCTION}

1.1. Motivation. Is Nature always looking for optimum for living organisms? In particular, are the organs designed to optimize some criterion? Complete answers to these questions are likely never to be discovered. Nevertheless, assuming that Nature proceeds in the most efficient way, can lead to a better understanding of the modeling of an organ and the underlying physical or chemical phenomena. This is this idea of inverse modeling that we had in mind when we began this work. Roughly speaking, it can be described by the following steps:

i Let us consider a given organ of a living body.

ii Write a mathematical model which describes the behavior of this organ.

iii Imagine a (numerical) criterion that Nature would like to optimize for this organ.

iv Determine the optimal shape for this criterion and this model.

v Compare with the real shape(s).

If the optimal and the real shapes coincide, we can guess that our model and our criterion are relevant. If they do not, we must admit that either our criterion or our model (or the initial guess that Nature looks for optimum) is probably wrong. We believe that it will often be the choice of the criterion which is not correct. A possible reason is the complexity of Nature. This complexity indicates that, in general, there is not a unique criterion to optimize but several ones (which could also be antagonists). The mathematical study (point 4 in the above procedure) becomes then much more difficult since one needs to use tools of multi-criteria optimization.

In this paper, we want to follow the above procedure in the case of a dendrite. We consider a fiber which is sealed at its right end and connected to a soma at its left end. We use the classical cable equation to describe the electrical potential along the fiber. What are the criterions that we can consider here? Of course, we want the dendrite to propagate the best as possible the electrical signal. In other terms, the attenuation of the signal must be as small as possible. We are going to consider the two kinds of possible attenuation: attenuation in time or in space and we are looking for the shape of a dendrite which minimizes this attenuation. In both cases, the optimal shape that we get is a cylinder. Since it is very close to the real shape, at first sight, we can conclude that Nature is in accordance with mathematics for this problem and solves a shape optimization problem! For a general

Date: 22nd May 2007.

2000 Mathematics Subject Classification. Primary 49J20; Secondary 49R50, 92C15.

Key words and phrases. optimal shape, cable equation, dendrite, eigenvalue problem.

Corresponding author: Antoine Henrot, Tel +33-383684560, Fax +33-383684534, E-mail address Antoine.Henrot@iecn.u-nancy.fr. 
reference in mathematical modeling in Neuroscience, we refer to the book of A. Scott, [15]. For a more exhaustive view and an introduction to the beauty of shapes (and, in particular, optimal shapes) in Nature, we refer to the classical books of S. Hildebrandt and A. Tromba, [9] and A. Bejan, [2].

1.2. The mathematical model. Let us consider a fiber with a cylindrical symmetry, of length $\ell$ and radius $a(x)$ at point $x$. We denote by $v(x, t)$ the difference from rest of the membrane potential at point $x$ and time $t$. The equation satisfied by $v(x, t)$ is similar to the classical cable equation as established by W. Rall during the sixties, cf [13], [14], [П]. See also, [16] as the best motivation of the current study. We consider here the case of a fiber which is sealed at its right end and connected to a soma with surface area $A_{s}$ at its left end. Let us denote by $R_{a}$ the axial resistance $(\mathrm{k} \Omega \mathrm{cm}), C_{m}$ is the membrane capacitance $\left(\mu \mathrm{F} / \mathrm{cm}^{2}\right), G_{m}$ the fiber membrane conductance and $G_{s}$ the soma membrane conductance $\left(\mathrm{mS} / \mathrm{cm}^{2}\right)$. We assume that the fiber is initially at rest and that it receives a transient current stimulus $i_{0}$ at the left. The parabolic equation satisfied by $v$ is then (see [4]):

$$
\left\{\begin{array}{lr}
\frac{1}{2 R_{a}} \frac{\partial}{\partial x}\left(a^{2} \frac{\partial v}{\partial x}\right)=a \sqrt{1+a^{\prime 2}}\left(C_{m} \frac{\partial v}{\partial t}+G_{m} v\right) & x \in(0, \ell), t>0 \\
\frac{\pi a^{2}(0)}{R_{a}} \frac{\partial v}{\partial x}(0, t)=A_{s}\left(C_{m} \frac{\partial v}{\partial t}(0, t)+G_{s} v(0, t)\right)-i_{0}(t) & t>0 \\
\frac{\partial v}{\partial x}(\ell, t)=0 & t>0 \\
v(x, 0)=0 & x \in(0, \ell) .
\end{array}\right.
$$

It is convenient to represent $v$, solution of (1), in terms of eigenfunctions as did S. Cox and J. Raol in [丹]:

$$
v(x, t)=\sum_{n=0}^{+\infty} \psi_{n}(t) \phi_{n}(x) \quad x \in(0, \ell), t>0
$$

where $\phi_{n}$ is the $n$-th eigenfunction associated to the eigenvalue $\mu_{n}$ :

$$
\left\{\begin{array}{l}
-\left(a^{2} \phi_{n}^{\prime}\right)^{\prime}=\mu_{n} a \sqrt{1+a^{\prime 2}} \phi_{n} \quad x \in(0, \ell) \\
\frac{2 \pi}{A_{s}} a^{2}(0) \phi_{n}^{\prime}(0)+\left(\mu_{n}+\gamma\right) \phi_{n}(0)=0 \\
\phi_{n}^{\prime}(\ell)=0
\end{array}\right.
$$

where $\gamma:=2 R_{a}\left(G_{m}-G_{s}\right)$ is assumed to be non negative. We choose to normalize the eigenfunctions by

$$
\left\|\phi_{n}\right\|_{a}^{2}:=A \phi_{n}^{2}(0)+\int_{0}^{\ell} a(x) \sqrt{1+a^{\prime 2}(x)} \phi_{n}^{2}(x) \mathrm{d} x=1 .
$$

where $A=\frac{A_{s}}{2 \pi}$. Of course the eigen-pair $\left(\mu_{n}, \phi_{n}\right)$ strongly depends on the taper $a(x)$ so we will often denote it by $\left(\mu_{n}(a), \phi_{n}(a)\right)$. The eigenvalue problem (3) is not classical since the eigenvalue appears in the boundary condition, see section 2 for more precisions.

Inserting the decomposition (2) in the equation (1) gives an ordinary differential equation satisfied by $\psi_{n}(t)$. After resolution, we get:

$$
v(x, t)=\frac{1}{2 \pi C_{m}} \sum_{n=1}^{+\infty} \phi_{n}(0) \phi_{n}(x) i_{0} * e^{-\lambda_{n} t},
$$

where $\lambda_{n}:=\frac{\mu_{n}+2 R_{a} G_{m}}{2 R_{a} C_{m}}>0$ (see Lemma 2.1) and $*$ denotes the convolution product of distributions.

1.3. The optimization problems. We need now to give a precise statement to the optimization problems presented in the introduction. In that purpose, we have to choose the functions we want to optimize and the class of functions $a(x)$ in competition. Let us begin 
with this last point. Since the fiber must not collapse, it is natural to assume a lower bound for the functions $a(x)$, so we fix a positive constant $a_{0}$ and we impose:

$$
\forall x \in[0, \ell], \quad a(x) \geq a_{0}>0 .
$$

Now the minimal regularity needed for $a$ is clearly, according to system (1) or (3) that the derivative $a^{\prime}$ exists (at least almost everywhere) and is bounded, so we choose to work in the class of Lipschitz continuous functions which is often denoted by $W^{1, \infty}(0, \ell)$. At last, we also need to put a constraint on the "cost" for Nature to build a fiber. It seems reasonable to consider that this cost is proportional to the surface area of the fiber. This surface area is clearly given by

$$
\text { Surface area }=2 \pi \int_{0}^{\ell} a(x) \sqrt{1+a^{\prime 2}(x)} \mathrm{d} x
$$

so we can assume a bound, say $S$, on this surface area. To summarize, we consider the class of functions $a(x)$ defined by:

$$
\mathcal{A}_{a_{0}, S}:=\left\{a \in W^{1, \infty}(0, \ell), a(x) \geq a_{0} \text { and } \int_{0}^{\ell} a(x) \sqrt{1+a^{\prime 2}(x)} \mathrm{d} x \leq S\right\} .
$$

Of course, we need to assume $S>a_{0} \ell$ in order that the class $\mathcal{A}_{a_{0}, S}$ be non trivial.

As explained in the Introduction, an "ideal" dendrite should conduct in the best possible way the electrical information he is supposed to transmit. In other terms, the attenuation of the signal must be minimized. Since the potential $v$ depends on the space and the time variable, we can consider both criterions.

1.3.1. Attenuation in space. Let us introduce the transfer function $T$ defined by :

$$
T(a):=\frac{\int_{0}^{+\infty} v(0, t) \mathrm{d} t}{\int_{0}^{+\infty} v(\ell, t) \mathrm{d} t} .
$$

$T(a)$ corresponds to the ratio of the mean values in time of the potential $v$ taken at points $x=0$ and $x=\ell$. This ratio is always greater than one, see Remark 3.1 and is a good indicator of the attenuation of the signal between the two extremities of the dendrite. So, it is realistic to look for a taper $a(x)$ which yields a ratio as close to one as possible:

Find $a$ in the class $\mathcal{A}_{a_{0}, S}$ which minimizes $T(a)$.

1.3.2. Attenuation in time. According to expansion (5), the potential $v(x, t)$ goes to 0 when $t \rightarrow+\infty$. More precisely, its asymptotic behavior is described by

$$
v(x, t) \simeq \frac{1}{2 \pi C_{m}} \phi_{1}(0) \phi_{1}(x) i_{0} * e^{-\lambda_{1} t}
$$

where $\lambda_{1}:=\frac{\mu_{1}(a)+2 R_{a} G_{m}}{2 R_{a} C_{m}}>0$ and $\mu_{1}(a)$ is the first eigenvalue of (3). Therefore, as it is classical in such parabolic problems, it seems natural to look for a function $a(x)$ which minimizes the exponential rate of decay:

$$
\text { Find } a \in \mathcal{A}_{a_{0}, S} \text { which minimizes } \mu_{1}(a) \text { (the first eigenvalue of (3)). }
$$

The idea of minimizing eigenvalues of such Sturm-Liouville operators is a long story and goes back at least to M. Krein in [10], see also [7] for a review on such problems.

1.3.3. The main result. We state in the following Theorem the main results of this paper

Theorem 1.1. $\quad$ i The unique minimizer of the eigenvalue $\mu_{1}(a)$ in the class $\mathcal{A}_{a_{0}, S}$ is the constant function $a \equiv a_{0}$.

ii The unique minimizer of the criterion $T(a)$ in the class $\mathcal{A}_{a_{0}, S}$ is the constant function $a \equiv a_{0}$. 
In other terms, for both criterions, the optimal shape of a dendrite sealed at one end and connected to a soma at the other end is the cylindrical one!

In his thesis and in a foregoing paper, see [11], [12], the second author studies the case of a dendrite sealed at both ends. From a mathematical point of view, it changes the boundary conditions in (1) and (3) which become homogeneous Neumann boundary conditions at both extremities. The result he obtains is the same for the case of attenuation in space, but it is different for the attenuation in time. Actually, there is no existence of a minimizer for $\mu_{1}(a)$ (as usual in the Neumann case $\mu_{0}(a)=0$ and $\mu_{1}(a)$ denotes the first non-zero eigenvalue). Moreover, he is able to exhibit minimizing sequences which would produce very strange dendrites!

1.4. Notation. The set of notation used in this paper is summarized in this section.

$W^{1, \infty}(0, \ell) \quad$ the set of Lipschitz continuous functions defined on the interval $[0, \ell]$.

$$
\begin{aligned}
& \text { the class of functions defined by } \\
& \left\{a \in W^{1, \infty}(0, \ell), a(x) \geq a_{0}, \int_{0}^{\ell} a(x) \sqrt{1+a^{\prime 2}(x)} \mathrm{d} x \leq S\right\} .
\end{aligned}
$$

$\|.\|_{\infty} \quad$ norm defined on the space of bounded functions $L^{\infty}(0, \ell)$ by $\|f\|_{\infty}=\sup _{t \in[0, \ell]}|f(t)|$.

inner product defined for two continuous functions $f$ and $g$ by: $<f, g>_{a}:=A f(0) g(0)+\int_{0}^{\ell} a(x) \sqrt{1+a^{\prime 2}(x)} f(x) g(x) \mathrm{d} x$.

$\|\cdot\|_{a} \quad$ norm induced by $<., .>_{a}$.

$\mathcal{E}_{a} \quad$ completion of the space of continuous functions $\mathcal{C}([0, \ell])$ for the norm $\|.\|_{a}$.

$L^{2}(0, \ell) \quad$ the space of (classes of) functions which are square-integrable on $(0, \ell)$.

$H^{1}(0, \ell) \quad$ the Sobolev space of functions in $L^{2}(0, \ell)$ whose derivative (in the sense of distributions) lies in $L^{2}(0, \ell)$.

$\left\langle\frac{\mathrm{d} J}{\mathrm{~d} \nu}\left(\nu_{0}\right), h\right\rangle \quad$ Gâteaux-derivative of a function $J$ at point $\nu_{0}$ in direction $h$ defined by: $\left\langle\frac{\mathrm{d} J}{\mathrm{~d} \nu}\left(\nu_{0}\right), h\right\rangle \stackrel{\text { def }}{=} \lim _{t \searrow 0} \frac{J\left(\nu_{0}+t h\right)-J\left(\nu_{0}\right)}{t}$.

\section{Minimization of The First EIGENVAlue}

The eigenvalue problem (3) is not completely classical due to the presence of the eigenvalue $\mu_{n}$ in the first boundary condition. As explained in [\#, see also the works of $\mathbf{J}$. Walter [17] and J. Ercolano-M. Schechter [5], a good way to handle with such case consists in introducing the following inner product:

$$
<f, g>_{a}=A f(0) g(0)+\int_{0}^{\ell} a(x) \sqrt{1+a^{\prime 2}(x)} f(x) g(x) \mathrm{d} x,
$$

its associated norm $\|.\|_{a}$ and the Hilbert space $\mathcal{E}_{a}$ defined as the completion of the space of continuous functions $\mathcal{C}([0, \ell])$ for this norm. It is easy to see that $\mathcal{E}_{a}$ is a space satisfying $H^{1}(0, \ell) \subset \mathcal{E}_{a} \subset L^{2}(0, \ell)$ (both inclusions are strict). Moreover, the map $\phi \mapsto \phi(0)$ defines a linear continuous form on $\mathcal{E}_{a}$. It is now classical spectral theory which allows to prove existence of a sequence of eigenvalues $\mu_{n}$ and eigenfunctions $\phi_{n}$ orthogonal for the inner product $<., .>_{a}$.

Let us now make an elementary observation on the sign of $\mu_{1}(a)$ : 
Lemma 2.1. Let a be in the class $\mathcal{A}_{a_{0}, S}$, then the first eigenvalue $\mu_{1}(a)$ of (3) satisfies

$$
-2 R_{a} G_{m}<-\gamma<\mu_{1}(a)<0 .
$$

Proof. Let $a \in \mathcal{A}_{a_{0}, S}$ and $v \in H^{1}(0, \ell)$, non identically zero. We denote by $R[a ; v]$, the Rayleigh quotient:

$$
R[a ; v]:=\frac{\int_{0}^{\ell} a^{2}(x) u^{\prime 2}(x) \mathrm{d} x-A \gamma u^{2}(0)}{\int_{0}^{\ell} a(x) \sqrt{1+a^{\prime 2}(x)} u^{2}(x) \mathrm{d} x+A u^{2}(0)} .
$$

The classical Poincaré-Courant-Hilbert principle writes:

$$
\mu_{1}(a)=\inf _{v \in H^{1}(0, \ell)} R[a ; v]
$$

Now, taking $v \equiv 1$ in the above formula yields:

$$
\mu_{1}(a) \leq-\frac{A \gamma}{\int_{0}^{\ell} a(x) \sqrt{1+a^{\prime 2}(x)} \mathrm{d} x+A}<0 .
$$

The lower bound is easy by observing that $\mu_{1}(a)+\gamma=R\left[a ; \phi_{1}(a)\right]+\gamma>0$.

Remark 2.1. Using the min-max formulae for the second eigenfunction, it is also possible to prove that the second eigenvalue satisfies $\mu_{2}(a)>0$. The proof consists in studying the problem of calculus of variations $\min \frac{1}{v^{2}(0)} \cdot \int_{0}^{\ell} a^{2}(x) v^{\prime 2}(x) \mathrm{d} x$ on the class $W_{a}:=\{v \in$ $H^{1}(0, \ell): v(0) \neq 0$ and $\left.<v, \phi_{1}(a)>_{a}=0\right\}$.

We can now prove the first part of Theorem 1.1. The eigenfunction $\phi_{1}(a)$ associated to $\mu_{1}(a)$ realizes the minimum of the Rayleigh quotient. Hence, we have:

$$
\mu_{1}(a)=\frac{\int_{0}^{\ell} a^{2}(x) \phi_{1}(a)^{\prime 2}(x) \mathrm{d} x-A \gamma \phi_{1}(a)^{2}(0)}{\int_{0}^{\ell} a(x) \sqrt{1+a^{\prime 2}(x)} \phi_{1}(a)^{2}(x) \mathrm{d} x+A \phi_{1}(a)^{2}(0)} .
$$

By Lemma 2.1, the numerator of this quotient is negative. Moreover, we have:

$$
\int_{0}^{\ell} a^{2}(x) \phi_{1}(a)^{\prime 2}(x) \mathrm{d} x-A \gamma \phi_{1}(a)^{2}(0) \geq a_{0}^{2} \int_{0}^{\ell} \phi_{1}(a)^{\prime 2}(x) \mathrm{d} x-A \gamma \phi_{1}(a)^{2}(0)
$$

and

$$
\frac{1}{\int_{0}^{\ell} a \sqrt{1+a^{\prime 2}} \phi_{1}(a)(x) \mathrm{d} x+A \phi_{1}(a)^{2}(0)} \leq \frac{1}{\int_{0}^{\ell} a_{0} \phi_{1}(a)(x) \mathrm{d} x+A \phi_{1}(a)^{2}(0)}
$$

(with a strict inequality in (13), (14) if $a$ is not constant). Finally, writing that $\mu_{1}\left(a_{0}\right)=$ $\inf _{v \in H^{1}(0, \ell)} R\left[a_{0} ; v\right]$, we deduce from (13), (14) and (12) that $\mu_{1}\left(a_{0}\right)<\mu_{1}(a)$ as soon as $a \neq a_{0}$.

\section{Minimization of $T(a)$}

3.1. Introduction. This last section is devoted to the proof of the second claim of Theorem 1.1, i.e. that $a=a_{0}$ minimizes the criterion $T(a)$. We recall that the criterion $T(a)$ describes the attenuation in space and that it is defined by

$$
T(a):=\frac{\int_{0}^{+\infty} v(0, t) \mathrm{d} t}{\int_{0}^{+\infty} v(\ell, t) \mathrm{d} t} .
$$


The proof here is much more complicated than for $\mu_{1}(a)$. Let us now outline the different steps of the proof.

1st step: Using the Laplace Transform $\widehat{v}(x, p)$ of $v(x, t)$, we rewrite the criterion $T(a)$ as the quotient $\widehat{v}(0,0) / \widehat{v}(\ell, 0)$.

2nd step: We use the change of variable defined by $y=\int_{0}^{x} \frac{\mathrm{d} t}{a^{2}(t)}$ to transform the equation satisfied by $\widehat{v}$ into a simpler differential equation. This allows us to consider a new unknown $\rho(y):=a^{3}(x) \sqrt{1+a^{\prime}(x)^{2}}$ instead of $a$ and a new criterion $T_{1}(\rho)$. The function $\rho$ must lie in the set defined by:

$$
\mathcal{R}_{a_{0}, S}:=\left\{\rho \in L^{\infty}\left(0, \ell_{1}\right): a_{0}^{3} \leq \rho(y) \text { and } \int_{0}^{\ell_{1}} \rho(y) \mathrm{d} y \leq S\right\} .
$$

3rd step: We solve the new optimization problem $\min T_{1}(\rho)$ first on the subclass of functions $\rho \in \mathcal{R}_{a_{0}, S}$ which satisfy $\rho \leq M$ for some positive constant $M$. We prove that the minimizer has to be a bang-bang function. It means that it can only takes the values $a_{0}^{3}$ and $M$. Then, studying carefully the optimality conditions, we prove that the only minimizer is $\rho \equiv a_{0}^{3}$.

4th step: We conclude.

3.2. Use of the Laplace Transform. The parabolic equation is not completely standard in the sense that it contains a dynamical boundary condition at $x=0$. This kind of problem has been studied by different people, see e.g. [6], [1] and the references therein. It can be proved that the solution $v(x, t)$ belongs to $L^{2}\left(0, T, H^{1}(0, \ell)\right)$. Moreover, using the eigenvalue expansion (2), one can see that, in the case of an impulsion $i_{0}=\delta$ (a Dirac measure at $t=0)$, the integrals $\int_{0}^{+\infty}|v(0, t)| \mathrm{d} t$ and $\int_{0}^{+\infty}|v(\ell, t)| \mathrm{d} t$ are well defined. Let us introduce the Laplace Transform $\widehat{v}(x, p)$ of the solution defined by

$$
\widehat{v}(x, p):=\int_{0}^{+\infty} e^{-p t} v(x, t) \mathrm{d} t .
$$

Thanks to the convergence of the integrals, the criterion $T(a)$ can be rewritten as

$$
T(a):=\frac{\int_{0}^{+\infty} v(0, t) \mathrm{d} t}{\int_{0}^{+\infty} v(\ell, t) \mathrm{d} t}=\frac{\lim _{p \rightarrow 0} \widehat{v}(0, p)}{\lim _{p \rightarrow 0} \widehat{v}(\ell, p)} .
$$

Now, transforming equation (11), we see that the Laplace Transform $\widehat{v}$ is the solution of the following o.d.e.:

$$
\begin{cases}\frac{1}{2 R_{a}} \frac{\partial}{\partial x}\left(a^{2} \frac{\partial \widehat{v}}{\partial x}\right)=a \sqrt{1+a^{\prime 2}}\left(C_{m} p \widehat{v}+G_{m} \widehat{v}\right) & (x, p) \in(0, \ell) \times(0 ;+\infty) \\ \frac{\pi a^{2}(0)}{R_{a}} \frac{\partial \widehat{v}}{\partial x}(0, p)=A_{s}\left[C_{m} p \widehat{v}(0, p)+G_{s} \widehat{v}(0, p)\right]-1 & p \in(0,+\infty) \\ \frac{\partial \widehat{v}}{\partial x}(\ell, p)=0 & p \in(0,+\infty) \\ \widehat{v}(x, 0)=0 & x \in(0, \ell) .\end{cases}
$$

3.3. A change of variable. We are now going to use the following change of variable classical in ordinary differential equations, see e.g. [3]

$$
y=\int_{0}^{x} \frac{\mathrm{d} t}{a^{2}(t)} .
$$

The interval $(0, \ell)$ becomes $\left(0, \ell_{1}\right)$ where

$$
\ell_{1}:=\int_{0}^{\ell} \frac{\mathrm{d} t}{a^{2}(t)}
$$

the function $\widehat{v}$ is transformed into the function

$$
w(y, p):=\widehat{v}(x, p) .
$$


and we consider a new unknown $\rho$ defined by :

$$
\rho(y):=a^{3}(x) \sqrt{1+a^{\prime}(x)^{2}} .
$$

Since $a$ belongs to the class $\mathcal{A}_{a_{0}, S}$ defined in (8), the new function $\rho$ belongs to:

$$
\mathcal{R}_{a_{0}, S}:=\left\{\rho \in L^{\infty}\left(0, \ell_{1}\right): a_{0}^{3} \leq \rho(y) \text { and } \int_{0}^{\ell_{1}} \rho(y) \mathrm{d} y \leq S\right\} .
$$

Then, equation (16) becomes:

$$
\begin{cases}\frac{1}{2 R_{a}} \frac{\partial^{2} w}{\partial y^{2}}=\rho\left(C_{m} p+G_{m}\right) w & (y, p) \in\left(0, \ell_{1}\right) \times(0 ;+\infty) \\ \frac{\pi}{R_{a}} \frac{\partial w}{\partial y}(0, p)=A_{s}\left[C_{m} p+G_{s}\right] w(0, p)-1 & p \in(0,+\infty) \\ \frac{\partial w}{\partial y}\left(\ell_{1}, p\right)=0 & p \in(0,+\infty) .\end{cases}
$$

We let $p$ going to 0 in the equation (19) (see Appendix $A$ for a mathematical justification) to get a function $w_{0}(y):=w(y, 0)$ which satisfies

$$
\begin{cases}\frac{1}{2 R_{a}} \frac{\mathrm{d}^{2} w_{0}}{\mathrm{~d} y^{2}}=\rho G_{m} w_{0} & y \in\left(0, \ell_{1}\right) \\ \frac{\pi}{R_{a}} \frac{\mathrm{d} w_{0}}{\mathrm{~d} y}(0)=A_{s} G_{s} w_{0}(0)-1 & \\ \frac{\mathrm{d} w_{0}}{\mathrm{~d} y}\left(\ell_{1}\right)=0 & \end{cases}
$$

Moreover, from (15) the criterion $T(a)$ becomes

$$
T(a)=T_{1}(\rho)=\frac{w_{0}(0)}{w_{0}\left(\ell_{1}\right)}
$$

The problems $\min \left\{T(a), a \in \mathcal{A}_{a_{0}, S}\right\}$ and $\min \left\{T_{1}(\rho), \rho \in \mathcal{R}_{a_{0}, S}\right\}$ are not completely equivalent since $a \in \mathcal{A}_{a_{0}, S} \mapsto \rho \in \mathcal{R}_{a_{0}, S}$ is not a one-to-one correspondance. Nevertheless, it is clear that $\mathcal{R}_{a_{0}, S}$ contains the whole image of $\mathcal{A}_{a_{0}, S}$ by this map. So, if we find a minimizer of $T_{1}$ in $\mathcal{R}_{a_{0}, S}$ which belongs to the image of $\mathcal{A}_{a_{0}, S}$ (this will be the case), we will solve our problem.

Remark 3.1. Let us have a look to equation (20). It is not possible that $w_{0}^{\prime}(0) \geq 0$ (otherwise $w_{0}(0)$ would be positive, according to the first boundary condition, and then $w_{0}$ would remain positive and convex which would contradict the second boundary condition. In the same way, $w_{0}(0)$ cannot be negative, otherwise $w_{0}$ would remain negative and concave and this is impossible with the second boundary condition. So finally, one can see that $w_{0}^{\prime}(0)<0, w_{0}(0)>0$ and $w_{0}$ remains positive on the whole interval $\left(0, \ell_{1}\right)$. At last, since $w_{0}^{\prime}$ is increasing and $w_{0}^{\prime}\left(\ell_{1}\right)=0$, we see that $w_{0}$ is decreasing (and positive), therefore $w_{0}\left(\ell_{1}\right)<w_{0}(0)$ which proves that $T(a)=T_{1}(\rho)<1$.

3.4. Study of a new optimization problem. Using the different transformations introduced in the previous subsections, we see that we must now solve the optimization problem: $\min T_{1}(\rho)$ with $\rho$ in the class $\mathcal{R}_{a_{0}, S}$. Let us begin by solving this optimization problem in the subclass

$$
\mathcal{R}_{a_{0}, S}^{M}:=\left\{\rho \in L^{\infty}\left(0, \ell_{1}\right): a_{0}^{3} \leq \rho(y) \leq M \text { and } \int_{0}^{\ell_{1}} \rho(y) \mathrm{d} y \leq S\right\}
$$

where $M$ is a fixed positive constant $\left(M>a_{0}^{3}\right)$. We will let $M \rightarrow+\infty$ later.

Theorem 3.1. The problem $\min T_{1}(\rho)$, with $\rho \in \mathcal{R}_{a_{0}, S}^{M}$, has a solution $\rho^{*}$. Moreover, every solution is a bang-bang function, i.e. a function which satisfies $\rho^{*}=a_{0}^{3}$ or $M$ almost everywhere.

Proof. The fact that the optimizer is a bang-bang function often occurs in such control problems. Existence of a minimizer $\rho^{*}$ is easy, due to continuity of the criterion $T_{1}(\rho)$ for the weak-* convergence. Then, we write and analyze the optimality conditions thanks to 
the introduction of two adjoint problems. We are able to prove that the set $\left\{a_{0}^{3}<\rho^{*}<M\right\}$ has zero measure. Let us now give the details.

Since $\mathcal{R}_{a_{0}, S}^{M}$ is a bounded subset of $L^{\infty}\left(0, \ell_{1}\right)$ it is compact for the weak-star convergence. So, to prove existence of a minimizer, we just need to prove that the criterion $T_{1}$ is continuous for the weak-star convergence. Let $\left(\rho_{n}\right)_{n \in \mathbb{N}}$ be a sequence in $\mathcal{R}_{a_{0}, S}^{M}$ such that $\rho_{n} \stackrel{*}{\rightarrow} \rho$ and let us denote by $w_{n}$ and $w$ the associated solutions of $(40)$. From the variational formulation of this problem

(22) $\frac{1}{2 R_{a}} \int_{0}^{\ell_{1}} w_{n}^{\prime}(y) z^{\prime}(y) \mathrm{d} y+G_{m} \int_{0}^{\ell_{1}} \rho_{n}(y) w_{n}(y) z(y) \mathrm{d} y+2 A G_{s} w_{n}(0) z(0)=\frac{z(0)}{2 \pi}$

for every $z \in H^{1}\left(0, \ell_{1}\right)$, we first see (taking $z=w_{n}$ in (22) that the sequence $w_{n}$ is bounded in $H^{1}\left(0, \ell_{1}\right)$. So, it converges (up to a subsequence) weakly in $H^{1}\left(0, \ell_{1}\right)$ and strongly in $L^{2}\left(0, \ell_{1}\right)$ to a function $w_{\infty}$. Now, these convergence are sufficient to pass to the limit in (22), so we have proved that $w_{\infty}=w$ and all the sequence converges since $w$ is the only accumulation point. Existence of a minimizer $\rho^{*}$ in the class $\mathcal{R}_{a_{0}, S}^{M}$ follows.

We want now to write the optimality conditions. Let $h \in W^{1, \infty}\left(0, \ell_{1}\right)$ be an admissible perturbation of the optimum. We will now denote by $\dot{w}_{0}$ the quantity:

$$
\dot{w}_{0}:=\left\langle\frac{\mathrm{d} w_{0}}{\mathrm{~d} \rho}\left(\rho^{*}\right), h\right\rangle .
$$

Classical variational analysis shows that $\dot{w}_{0}$ is the solution of the ordinary differential equation:

$$
\left\{\begin{array}{l}
\frac{1}{2 R_{a}} \frac{\mathrm{d}^{2} \dot{w}_{0}}{\mathrm{~d} y^{2}}=G_{m}\left(\rho \dot{w_{0}}+h w_{0}\right) \quad y \in\left(0, \ell_{1}\right) \\
\frac{\pi}{R_{a}} \frac{\mathrm{d} \dot{w}_{0}}{\mathrm{~d} y}(0)=A_{s} G_{s} \dot{w}_{0}(0) \\
\frac{\mathrm{d} \dot{w}_{0}}{\mathrm{~d} y}\left(\ell_{1}\right)=0 .
\end{array}\right.
$$

Differentiating the criterion $T_{1}$ in the direction $h$ gives:

$$
\left\langle\frac{\mathrm{d} T_{1}}{\mathrm{~d} \rho}, h\right\rangle=\frac{\dot{w}_{0}(0) w_{0}\left(\ell_{1}\right)-w_{0}(0) \dot{w}_{0}\left(\ell_{1}\right)}{w_{0}^{2}\left(\ell_{1}\right)} .
$$

Let us now introduce the two following adjoint problems. We consider the function $q_{1}$ defined as the solution of the ordinary differential equation:

$$
\left\{\begin{array}{l}
\frac{1}{2 R_{a}} \frac{\mathrm{d}^{2} q_{1}}{\mathrm{~d} y^{2}}=G_{m} \rho\left(q_{1}(y)-y\right) \quad y \in\left(0, \ell_{1}\right) \\
\frac{\pi}{R_{a}} \frac{\mathrm{d} q_{1}}{\mathrm{~d} y}(0)=A_{s} G_{s} q_{1}(0) \\
\frac{\mathrm{d} q_{1}}{\mathrm{~d} y}\left(\ell_{1}\right)=0
\end{array}\right.
$$

and the function $q_{2}$ solution of :

$$
\left\{\begin{array}{l}
\frac{1}{2 R_{a}} \frac{\mathrm{d}^{2} q_{2}}{\mathrm{~d} y^{2}}=G_{m} \rho\left(q_{2}(y)-1\right) \quad y \in\left(0, \ell_{1}\right) \\
\frac{\pi}{R_{a}} \frac{\mathrm{d} q_{2}}{\mathrm{~d} y}(0)=A_{s} G_{s} q_{2}(0) \\
\frac{\mathrm{d} q_{2}}{\mathrm{~d} y}\left(\ell_{1}\right)=0
\end{array}\right.
$$

(existence and uniqueness of $q_{1}, q_{2}$ follows from Lax-Milgram Theorem). Multiplying equation (23) by $q_{2}-1$, equation (26) by $\dot{w}_{0}$ and integrating both by parts yields:

$$
\dot{w}_{0}(0)=\frac{G_{m}}{A G_{s}} \int_{0}^{\ell_{1}} h(y) w_{0}(y)\left(q_{2}(y)-1\right) d y .
$$

In the same way, multiplying equation (23) by $q_{1}-y$, equation (25) by $\dot{w}_{0}$ and integrating both by parts yields:

$$
\dot{w}_{0}\left(\ell_{1}\right)=\dot{w}_{0}(0)+2 R_{a} G_{m} \int_{0}^{\ell_{1}} h(y) w_{0}(y)\left(q_{1}(y)-y\right) d y .
$$


Therefore, (24) together with $(27)$ and $(28)$ gives

$\left\langle\frac{\mathrm{d} T_{1}}{\mathrm{~d} \rho}, h\right\rangle=\frac{2 R_{a} G_{m}}{w_{0}\left(\ell_{1}\right)^{2}} \int_{0}^{\ell_{1}} h(y) w_{0}(y)\left(\frac{w_{0}\left(\ell_{1}\right)-w_{0}(0)}{\tilde{A}}\left(q_{2}(y)-1\right)-w_{0}(0)\left(q_{1}(y)-y\right)\right) \mathrm{d} y$

where $\tilde{A}=2 A G_{s} R_{a}$. Let us denote by $f$, the function of one variable defined by:

$$
\begin{aligned}
f:\left[0, \ell_{1}\right] & \longrightarrow \mathbb{R} \\
y & \longmapsto \frac{2 R_{a} G_{m}}{w_{0}\left(\ell_{1}\right)^{2}}\left[\frac{w_{0}\left(\ell_{1}\right)-w_{0}(0)}{\tilde{A}}\left(q_{2}(y)-1\right)-w_{0}(0)\left(q_{1}(y)-y\right)\right] .
\end{aligned}
$$

So, finally:

$$
\left\langle\frac{\mathrm{d} T_{1}}{\mathrm{~d} \rho}, h\right\rangle=\int_{0}^{\ell_{1}} h(y) w_{0}(y) f(y) \mathrm{d} y .
$$

We want now to prove that the optimum $\rho^{*}$ is a bang-bang function. It is a classical approach, see e.g. [8]. For that purpose, let us introduce the following sets:

- $\mathcal{I}_{0}\left(\rho^{*}\right):=\left\{y \in\left(0, \ell_{1}\right): \rho^{*}(y)=a_{0}\right\}$;

- $\mathcal{I}_{M}\left(\rho^{*}\right):=\left\{y \in\left(0, \ell_{1}\right): \rho^{*}(y)=M\right\}$;

- $\mathcal{I}_{\star}\left(\rho^{*}\right):=\left(\left\{y \in\left(0, \ell_{1}\right): a_{0}<\rho^{*}(y)<M\right\}\right.$.

We write $\mathcal{I}_{\star}\left(\rho^{*}\right)=\bigcup_{k=1}^{+\infty}\left\{y \in\left(0, \ell_{1}\right): a_{0}+\frac{1}{k}<\rho^{*}(y)<M-\frac{1}{k}\right\}=\bigcup_{k=1}^{+\infty} \mathcal{I}_{\star, k}\left(\rho^{*}\right)$. We want to prove that $\mathcal{I}_{\star, k}\left(\rho^{*}\right)$ has zero measure, for all integer $k \neq 0$. We argue by contradiction. Let us suppose that $\left|\mathcal{I}_{\star, k}\left(\rho^{*}\right)\right| \neq 0$. Let $y_{0} \in \mathcal{I}_{\star, k}\left(\rho^{*}\right)$. We denote by $\left(G_{k, n}\right)_{n \geq 0}$, the sequence of subsets of $\mathcal{I}_{\star, k}$ :

$$
G_{k, n}:=B\left(y_{0}, \frac{1}{n}\right) \cap \mathcal{I}_{\star, k}\left(\rho^{*}\right) \subset \mathcal{I}_{\star, k}\left(\rho^{*}\right) .
$$

Let us notice that $\bigcap_{n=0}^{+\infty} G_{k, n}=\left\{y_{0}\right\}$, and let us choose $h=\chi_{G_{k, n}}$. Then, for $t$ small enough, perturbations $\rho^{*}+t h$ et $\rho^{*}-t h$ are admissible. Then:

$$
\lim _{t \searrow 0} \frac{T_{1}\left(\rho^{*}+t h\right)-T_{1}\left(\rho^{*}\right)}{t}=\int_{0}^{\ell} h(y) w_{0}(y) f(y) \mathrm{d} y \geq 0 \Longleftrightarrow \int_{G_{k, n}} w_{0}(y) f(y) \mathrm{d} y \geq 0 .
$$

In the same way:

$\lim _{t \searrow 0} \frac{T_{1}\left(\rho^{*}-t h\right)-T_{1}\left(\rho^{*}\right)}{t}=-\int_{0}^{\ell} h(y) w_{0}(y) f(y) \mathrm{d} y \geq 0 \Longleftrightarrow \int_{G_{k, n}} w_{0}(y) f(y) \mathrm{d} y \leq 0$.

We can deduce that $\int_{G_{k, n}} w_{0}(y) f(y) \mathrm{d} y=0$. We divide by $\left|G_{k, n}\right|$ and we make $n$ tending to $+\infty$. The Lebesgue density theorem shows that $w\left(y_{0}\right) f\left(y_{0}\right)=0$, a.e. for $y_{0} \in \mathcal{I}_{\star, k}\left(\rho^{*}\right)$. This is clearly a contradiction, since $w_{0}$ and $f$ are respectively solutions of the differential equations $\frac{1}{2 R_{a}} \frac{\mathrm{d}^{2} w_{0}}{\mathrm{~d} y^{2}}=\rho^{*} G_{m} w_{0}$ and $\frac{1}{2 R_{a}} \frac{\mathrm{d}^{2} f}{\mathrm{~d} y^{2}}=\rho^{*} G_{m} f$. This proves that $\left|\mathcal{I}_{\star, k}\left(\rho^{*}\right)\right|=$ 0 and then $\mathcal{I}_{\star}\left(\rho^{*}\right)$ has also zero measure, what implies that $\rho^{*}$ equals $a_{0}^{3}$ or $M$ almost everywhere.

Now, we prove that among every bang-bang function, this is the constant function $a_{0}^{3}$ which yields the minimum of $T_{1}$.

Lemma 3.1. The optimum of $T_{1}$ in the class $\mathcal{R}_{a_{0}, S}^{M}$ is the constant function $\rho(y)=a_{0}^{3}$.

Proof. Using notation of the proof of Theorem 3.1, the optimality conditions writes

- On the set $\mathcal{I}_{0}\left(\rho^{*}\right)$, we have $f(y) \geq 0$ and $h(y) \geq 0$;

- On the set $\mathcal{I}_{M}\left(\rho^{*}\right)$, we have $f(y) \leq 0$ and $h(y) \leq 0$. 
According to the differential equation (26) and maximum principle, the function $q_{2}-1$ is negative on $\left[0, \ell_{1}\right]$. Let us write $f$ like below:

$$
f(y)=\frac{2 R_{a} G_{m}}{w_{0}\left(\ell_{1}\right)^{2}}\left(q_{2}(y)-1\right) \cdot\left(\frac{w_{0}\left(\ell_{1}\right)-w_{0}(0)}{\tilde{A}}-w_{0}(0) g(y)\right),
$$

where $g(y):=\frac{q_{1}(y)-y}{q_{2}(y)-1}$. The function $g$ is two times derivable on $\left(0, \ell_{1}\right)$, and, using equations (25), (26) we have:

$$
\forall y \in\left(0, \ell_{1}\right), \frac{\mathrm{d}^{2} g}{\mathrm{~d} y^{2}}(y)=-2 \frac{\frac{\mathrm{d}}{\mathrm{d} y}\left[q_{2}-1\right](y)}{q_{2}(y)-1} \cdot \frac{\mathrm{d} g}{\mathrm{~d} y}(y) .
$$

Therefore, on every interval where $\frac{\mathrm{d} g}{\mathrm{~d} y}$ keeps his sign, we have:

$$
\frac{\mathrm{d} g}{\mathrm{~d} y}(y)=\frac{C}{\left[q_{2}(y)-1\right]^{2}}, \text { with } C \in \mathbb{R} .
$$

Now, $\frac{\mathrm{d} g}{\mathrm{~d} y}$ is continuous on $\left[0, \ell_{1}\right]$, then the only possibility is:

$$
\forall y \in\left(0, \ell_{1}\right), \frac{\mathrm{d} g}{\mathrm{~d} y}(y)=-\frac{q_{2}\left(\ell_{1}\right)-1}{\left[q_{2}(y)-1\right]^{2}}>0 .
$$

It follows that $g$ is an increasing function on $\left[0, \ell_{1}\right]$. Since $q_{2}-1$ is negative and $w_{0}(0)>0$, we see that: $f(y) \geq 0 \Longleftrightarrow g(y) \geq \frac{w\left(\ell_{1}\right)-w(0)}{\tilde{A} w(0)}$. Then, according to the optimality conditions the function $\rho^{*}$, local optimum for the criterion $T_{1}$, is necessarily as follows:

$$
\rho^{*}(y)= \begin{cases}M & \text { if } y<\xi_{1} \\ a_{0}^{3} & \text { if } y>\xi_{1}\end{cases}
$$

with a transition point $\xi_{1}$ which is possibly 0 or $\ell_{1}$. Now, it remains to look for the better

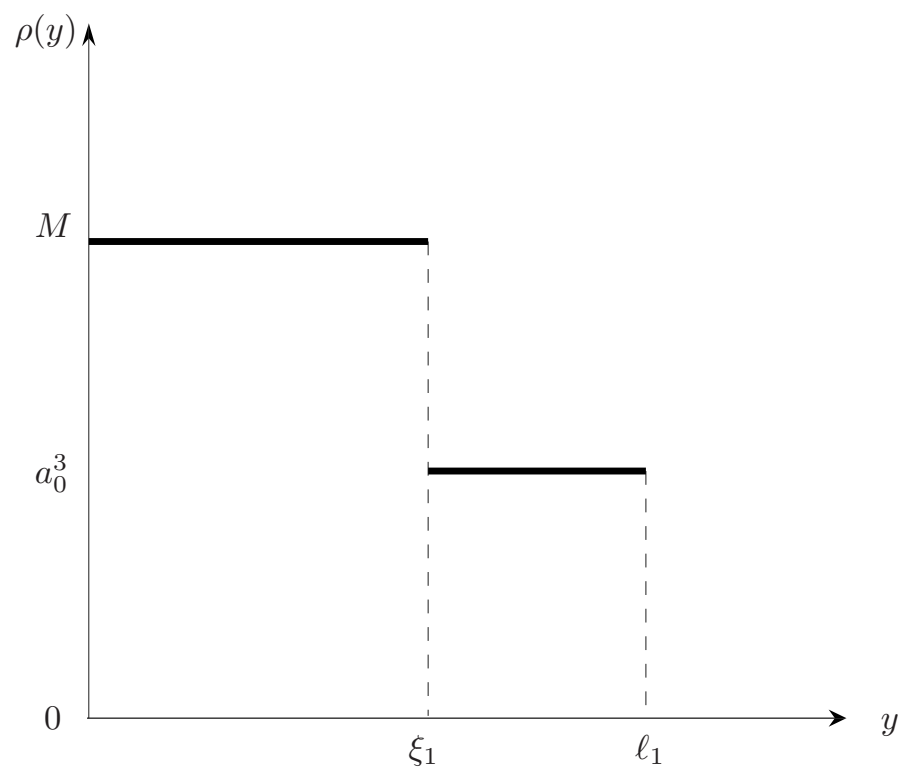

FIGURE 1. Possible profile of the optimum

$\rho^{*}$ among all functions defined by $(32$ ). The only unknown is finally the transition point 
$\xi_{1}$. We can use the explicit expression of the solution $w_{0}$ of equation (40) for such a simple $\rho^{*}$. We write $w_{0}$

$$
w_{0}(y)= \begin{cases}\alpha_{1}^{M} \cosh \left(\omega_{M} y\right)+\alpha_{2}^{M} \sinh \left(\omega_{M} y\right) & \text { on }\left[0, \xi_{1}\right] \\ \alpha_{3}^{M} \cosh \left(\omega_{0} y\right)+\alpha_{4}^{M} \sinh \left(\omega_{0} y\right) & \text { on }\left[\xi_{1}, \ell_{1}\right]\end{cases}
$$

where $\alpha_{1}^{M}, \alpha_{2}^{M}, \alpha_{3}^{M}$ and $\alpha_{4}^{M}$ are four constants and $\omega_{0}:=\sqrt{2 R_{a} G_{m} a_{0}^{3}}, \omega_{M}:=\sqrt{2 R_{a} G_{m} M}$. Thanks to boundary conditions, we get

$$
w_{0}(y)= \begin{cases}\alpha_{1}^{M} \cosh \left(\omega_{M} y\right)+\frac{A_{s} G_{s} \alpha_{1}^{M}-1}{\frac{\pi}{R_{a}} \omega_{M}} \sinh \left(\omega_{M} y\right) & \text { on }\left[0, \xi_{1}\right] \\ \alpha_{3}^{M}\left(\cosh \left(\omega_{0} y\right)-\tanh \left(\omega_{0} \ell_{1}\right) \sinh \left(\omega_{0} y\right)\right) & \text { on }\left[\xi_{1}, \ell_{1}\right]\end{cases}
$$

Therefore, the criterion $T_{1}$ is given by

$$
T_{1}\left(\rho^{*}\right)=\frac{w_{0}(0)}{w_{0}\left(\ell_{1}\right)}=\frac{\alpha_{1}^{M}}{\alpha_{3}^{M}} \cosh \left(\omega_{0} \ell_{1}\right) .
$$

Finally, using the fact that $w_{0}$ is $C^{1}$, we get, thanks to continuity of $w_{0}$ and $\frac{d w_{0}}{d y}$ at $y=\xi_{1}$

$$
\begin{array}{ll}
\frac{\alpha_{1}^{M}}{\alpha_{3}^{M}}= & \cosh \left(\omega_{0} \xi_{1}\right) \cosh \left(\omega_{M} \xi_{1}\right)-\frac{\omega_{0}}{\omega_{M}} \sinh \left(\omega_{M} \xi_{1}\right) \sinh \left(\omega_{0} \xi_{1}\right) \\
35) \quad & -\tanh \left(\omega_{0} \ell_{1}\right)\left[\sinh \left(\omega_{0} \xi_{1}\right) \cosh \left(\omega_{M} \xi_{1}\right)-\frac{\omega_{0}}{\omega_{M}} \sinh \left(\omega_{M} \xi_{1}\right) \cosh \left(\omega_{0} \xi_{1}\right)\right]
\end{array}
$$

and then

$$
\begin{array}{ll}
T_{1}\left(\rho^{*}\right)= & \cosh \left(\omega_{0} \ell_{1}\right)\left[\cosh \left(\omega_{0} \xi_{1}\right) \cosh \left(\omega_{M} \xi_{1}\right)-\frac{\omega_{0}}{\omega_{M}} \sinh \left(\omega_{M} \xi_{1}\right) \sinh \left(\omega_{0} \xi_{1}\right)\right] \\
\text { (36) } \quad-\sinh \left(\omega_{0} \ell_{1}\right)\left[\sinh \left(\omega_{0} \xi_{1}\right) \cosh \left(\omega_{M} \xi_{1}\right)-\frac{\omega_{0}}{\omega_{M}} \sinh \left(\omega_{M} \xi_{1}\right) \cosh \left(\omega_{0} \xi_{1}\right)\right]
\end{array}
$$

Computing the derivative of the criterion with respect to the variable $\xi_{1}$, we have

$$
\frac{\mathrm{d} T_{1}\left(\rho^{*}\right)}{\mathrm{d} \xi_{1}}=\left(\frac{\omega_{M}^{2}-\omega_{0}^{2}}{\omega_{M}}\right) \sinh \left(\omega_{M} \xi_{1}\right) \cosh \left[\omega_{0}\left(\ell_{1}-\xi_{1}\right)\right] \geq 0 .
$$

It follows that $\xi_{1}$ has to be equal to 0 , that means that the constant function $a_{0}^{3}$ minimize the criterion $T_{1}$ on $\mathcal{R}_{a_{0}, S}^{M}$.

3.5. Conclusion. The proof of Theorem 1.1 follows now easily. Since $a_{0}^{3}$ is the unique minimizer of the criterion $T_{1}$ in the class $\mathcal{R}_{a_{0}, S}^{M}$ and

$$
\mathcal{R}_{a_{0}, S}=\bigcup_{M>a_{0}^{3}} \mathcal{R}_{a_{0}, S}^{M} .
$$

we get that $a_{0}^{3}$ is the (unique) minimizer of $T_{1}$ in the class $\mathcal{R}_{a_{0}, S}$. Moreover, since $a_{0}^{3}=$ $a_{0}^{3} \sqrt{1+\left(\frac{\mathrm{d} a_{0}}{\mathrm{~d} y}\right)^{2}}$, it is clear that $T_{1}\left(a_{0}^{3}\right)=T\left(a_{0}\right)$ and then, $a_{0}$ minimizes $T$ on the class $\mathcal{A}_{a_{0}, S}$.

\section{ApPendix A. Limit OF $w_{p}$ WHEN $p \rightarrow 0$}

Let us now denote by $w_{p}$ the function $w(., p)$ for a given positive $p$. The equation 197 can be rewritten:

$$
\left\{\begin{array}{l}
\frac{1}{2 R_{a}} \frac{\mathrm{d}^{2} w_{p}}{\mathrm{~d} y^{2}}=\rho\left(C_{m} p+G_{m}\right) w_{p} \quad y \in\left(0, \ell_{1}\right) \\
\frac{\pi}{R_{a}} \frac{\mathrm{d} w_{p}}{\mathrm{~d} y}(0)=A_{s}\left[C_{m} p+G_{s}\right] w_{p}(0)-1 \\
\frac{\mathrm{d} w_{p}}{\mathrm{~d} y}\left(\ell_{1}\right)=0
\end{array}\right.
$$


We recall that we want to prove that $w_{p}$ has a limit $w_{0}$ when $p \rightarrow 0$ and that $w_{0}$ is the solution of (20). We can suppose that $p \in(0,1]$. Let us write the variational formulation of $(38)$ :

with

$$
\forall z \in H^{1}\left(0, \ell_{1}\right), a_{p}\left(w_{p}, z\right)=L(z)
$$

$a_{p}(u, z)=\frac{1}{2 R_{a}} \int_{0}^{\ell_{1}} u^{\prime}(y) z^{\prime}(y)+\left(C_{m} p+G_{m}\right) \rho(y) u(y) z(y) \mathrm{d} y+A\left(C_{m} p+G_{s}\right) u(0) z(0)$

and

$$
L(z)=\frac{z(0)}{2 \pi}
$$

Let us make $z=w_{p}$ in the above formulation. Since $a_{p}$ is uniformly (with respect to $p$ ) coercive:

$$
\min \left(\frac{1}{2 R_{a}}, a_{0}^{3} G_{m}\right)\left\|w_{p}\right\|_{H^{1}\left(0, \ell_{1}\right)}^{2} \leq a_{p}\left(w_{p}, w_{p}\right)=L\left(w_{p}\right)
$$

and $L$ is a linear continuous form, we get from (39) that the sequence $w_{p}$ is bounded in $H^{1}\left(0, \ell_{1}\right)$. Therefore, there exists $w^{*} \in H^{1}\left(0, \ell_{1}\right)$ such that $w_{p} \underset{p \rightarrow 0}{\rightarrow} w^{*}$ in $H^{1}\left(0, \ell_{1}\right)$ and $w_{p} \underset{p \rightarrow 0}{\longrightarrow} w^{*}$ in $L^{2}\left(0, \ell_{1}\right)$ (up to a subsequence). It follows that

- $w_{p}(0) \underset{p \rightarrow 0}{\longrightarrow} w^{*}(0)$,

- $\int_{0}^{\ell_{1}} \rho(y) w_{p}(y) z(y) \mathrm{d} y \underset{p \rightarrow 0}{\longrightarrow} \int_{0}^{\ell_{1}} \rho(y) w^{*}(y) z(y) \mathrm{d} y$

- $\int_{0}^{\ell_{1}} w_{p}^{\prime}(y) z(y) \mathrm{d} y \underset{p \rightarrow 0}{\longrightarrow} \int_{0}^{\ell_{1}} w^{* \prime}(y) z^{\prime}(y) \mathrm{d} y$.

Therefore $w^{*}$ is the solution of the ordinary differential equation:

$$
\left\{\begin{array}{l}
\frac{1}{2 R_{a}} \frac{\mathrm{d}^{2} w_{0}}{\mathrm{~d} y^{2}}=\rho G_{m} w_{0} \\
\frac{\pi}{R_{a}} \frac{\mathrm{d} w_{0}}{\mathrm{~d} y}(0)=A_{s} G_{s} w_{0}(0)-1 \\
\frac{\mathrm{d} w_{0}}{\mathrm{~d} y}\left(\ell_{1}\right)=0 .
\end{array}\right.
$$

which means that $w^{*}=w_{0}$, the desired result.

\section{REFERENCES}

[1] C. Bandle, J. Von Below, W. Reichel, Parabolic problems with dynamical boundary conditions: eigenvalue expansions and blow-up, Rend. Lincei Mat. Appl. 17 (2006), 35-67.

[2] A. BEJAN, Shape and Structure, from engineering to Nature Cambridge University Press, (2000).

[3] S.J. Cox, R. Lipton, Extremal eigenvalue problems for two-phase conductors, Arch. Rational Mech. Anal. 136 (1996), 101-117.

[4] S.J. CoX, J.H. RAOL, Recovering the passive properties of tapered dendrites from single and dual potential recordings, Math. Biosci. 190 (2004), no. 1, 9-37.

[5] J. ERCOLANO, M. SCHECHTER, Spectral theory for operators generated by elliptic boundary problems with eigenvalue parameter in boundary conditions, Comm. Pure Appl. Math. 18 (1965), 83-105.

[6] J. EsCHER Quasilinear parabolic systems with dynamical boundary conditions, Comm. Partial Diff. Equations 18 (1993), 1309-1364.

[7] A. HenRot Extremum problems for eigenvalues of elliptic operators, Frontiers in Mathematics, Birkhäuser (2006).

[8] A. Henrot, H. Maillot Optimization of the shape and the location of the actuators in an internal control problem, Boll. Unione Mat. Ital. Sez. B Artic. Ric. Mat. 84 (2001), no. 3, 737-757.

[9] S. Hildebrandt, A. Tromba The parsimonious universe, shape and form in the natural world, SpringerVerlag, New York, 1996.

[10] M.G. KREIN, On certain problems on the maximum and minimum of characteristic values and on the Lyapunov zones of stability, Amer. Math. Soc. Transl. (2) 1 (1955), 163-187.

[11] Y PRIVAT, phD thesis of the University of Nancy.

[12] Y PRIVAT, The optimal shape of a dendrite sealed at both ends. to appear

[13] W. RALL Theory of physiological properties of dendrites, Ann, NY Acad Sciences 96 (1962) 1071. 
[14] W. Rall, H. Agmon-Snir Cable theory for dendritic neurons, C. Koch, I. Segev (Eds) Methods in Neuronal Modeling second edition, MIT, Cambridge, MA, 1998.

[15] A. ScotT Neuroscience: A Mathematical Primer, Springer-Verlag, New York, 2002.

[16] G. StUART, N. SPRUSTON Determinants of voltage attenuation in neocortical pyramidal neuron dendrites, J. Neurosci. 1810 (1998), 3501-3510.

[17] J WALTER, Regular eigenvalue problem with eigenvalue parameter in the boundary condition, Math. Z. 133 (1973), 301-312.

InSTITUt ÉLIE CARTAN DE NANCY, UMR 7502 NANCY-UNIVERSITÉ - CNRS - INRIA, B.P. 239, VANDEUVRELÈS-NANCY CEDEX FRANCE 